\title{
CHARACTERISTICS OF POSITIVE BLOOD CULTURES, ANTIBIOTIC THERAPY AND OUTCOMES IN PAEDIATRIC BONE MARROW TRANSPLANT RECIPIENTS - A RETROSPECTIVE OBSERVATIONAL STUDY
}

L. Zombori' ${ }^{1}$, S. Ramachandrakurup ${ }^{1}$, L. Karnik ${ }^{1,2}$, J. de la Fuente ${ }^{1,2}$, J. Hatcher ${ }^{3}$.

${ }_{1}$ Imperial College Healthcare NHS Trust, Department of Paediatrics- St. Mary's Hospital, London, United Kingdom.

2Imperial College Healthcare NHS Trust, Centre for Haematology- Imperial College London, London, United Kingdom.

${ }^{3}$ Imperial College Healthcare NHS Trust, Department of Infection, London, United Kingdom

\section{Background}

Bone marrow transplantation (BMT) recipients are particularly susceptible to systemic infections. We aimed to characterize organisms leading to bacteriaemia in this group and map this to clinical data. In a retrospective observationa study we analyzed the data of all post BMT patients with a positive blood culture to reflect on our current choice of antibiotics and removal of central lines.

\section{Organisms}

Gram positive 8 coagulase negative Staphylococcus 2 Staphylococcus aureus 1 beta haemolytic Streptococcus group $G$ 1 Streptococcus sanguinis 1 Streptococcus mitis

1 Vancomycin resistant Enterococcus 1 Enterococcus faecium 1 Micrococcus sp.

Gram negative

4 Klebsiella pneumoniae 2 Klebsiella oxytoca 2 Enterobacter cloacae 2 Escherichia coli

1 Pseudomonas aeruginosa 1 Acinetobacter $s p$

3 Stenotrophomonas maltophilia 2 Ochrobactrum anthropii

\section{Yeast}

2 Candida glabrata

\section{Methods}

Positive blood cultures were identified retrospectively from laboratory data between the period of February 2016 to March 2017 in a large paediatric BMT centre in London.

Clinical data included time of positivity compared to transplantation date, type of transplant, neutropaenia, presence of a central venous catheter (CVC), community or hospital onset, type and length of antibiotic treatment clinical outcome and resistance patterns.

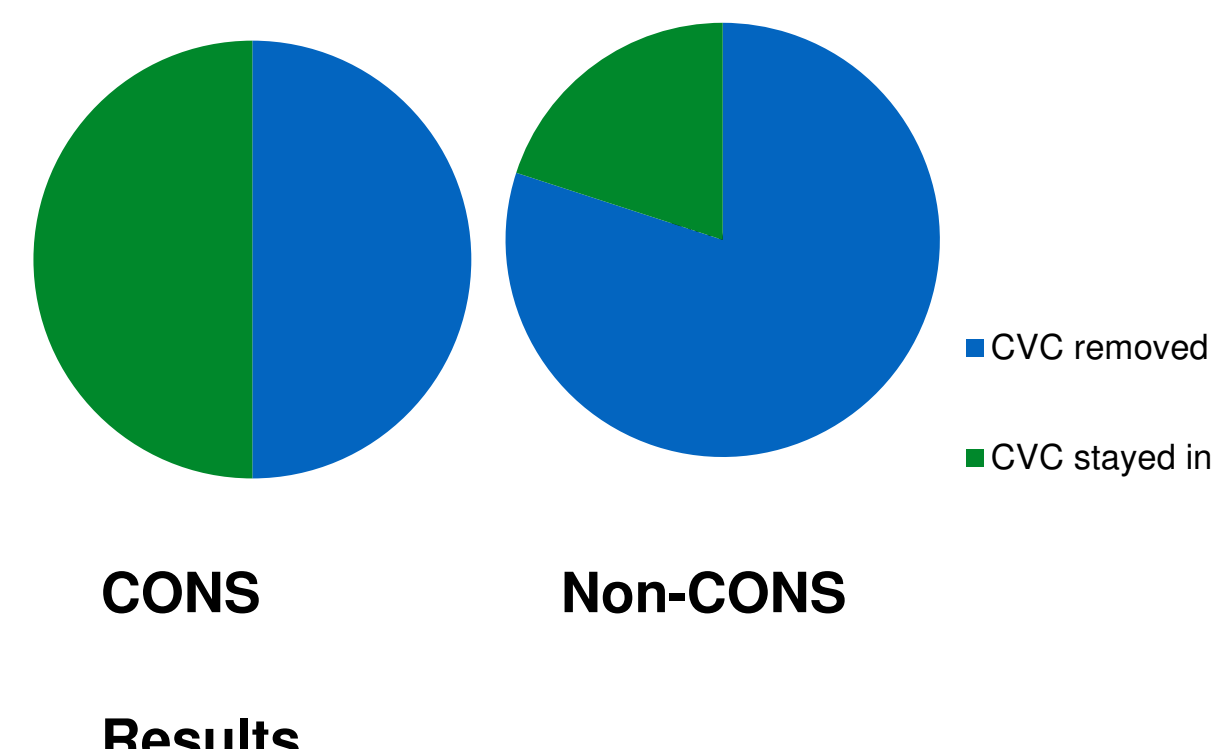

35 positive blood cultures from 28 clinical 35 positive blood cultures
episodes were identified.

Not surprisingly, most of the patients, $68 \%$ presented in the first 90 days post transplantation. In the first three weeks every patient was neutropenic, and even in the first 3 months, $79 \%$ were neutropenic

An indwelling CVC was present in $89 \%$ of episodes, in other words, all patients until 150 days, and no patients over 150 days post transplant had a central line.

$64 \%(18 / 28)$ were hospital onset episodes.

We thought having a haploidentical transplant will have a protective value against infections given the decreased need for immunosuppression, but exactly half of the episodes came from haplo transplant recipients.
The organisms found in the 3 episodes without a CVC were a Stenotrophomonas, a $S$. sanguinis, and a CONS, the last one in a patient who was surprisingly neutropenic at 266 days post transplant.

Coagulase negative Staphylococci (CONS) were the most common pathogen in $28 \%$., followed by Klebsiella pneumoniae (14\%) and Stenotrophomonas maltophilia (10\%)

$18 \%$ of the tested organisms were resistant to our first line piperacillin/tazobactam., but only $4 \%$ to Ciprofloxacin, our neutropenic prophylactic agent. All tested organisms were sensitive to Gentamicin and Amikacin, both of which drugs we use as back-up. We had a $20 \%$ Vancomycin resistance rate (1 VRE), but all tested organsims were sensitive to Meropenem and Teicoplanin.

$50 \%$ of CONS, $80 \%$ of non-CONS CVCs were removed within 7 days of bacteriaemia. $95 \%$ of CVC tips were cultured but only 1 cultured the organism same as the blood (a Klebsiella pneumoniae)

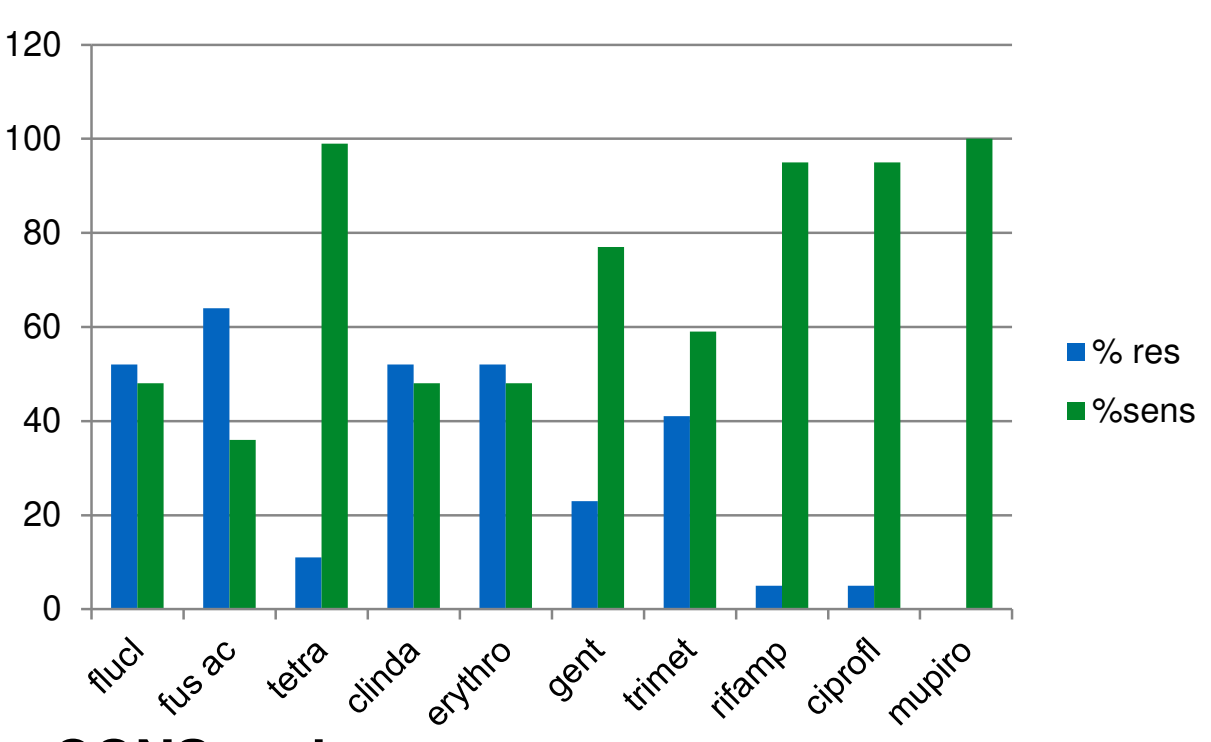
CONS resistance

\section{Conclusions}

Bacteriaemias are common complications of BMT. Identifying the responsible organisms allows targeted therapy and surveillance of regimens. We need to monitor the percentage of resistance to antibiotics and review our guidelines. We had a high rate of CVC removal with a low rate of positive line tip culture. Further study is needed as to whether early removal of CVCs in all bacteraemic patients is necessary.

The 30 day mortality rate was $14 \%$.

No of episodes

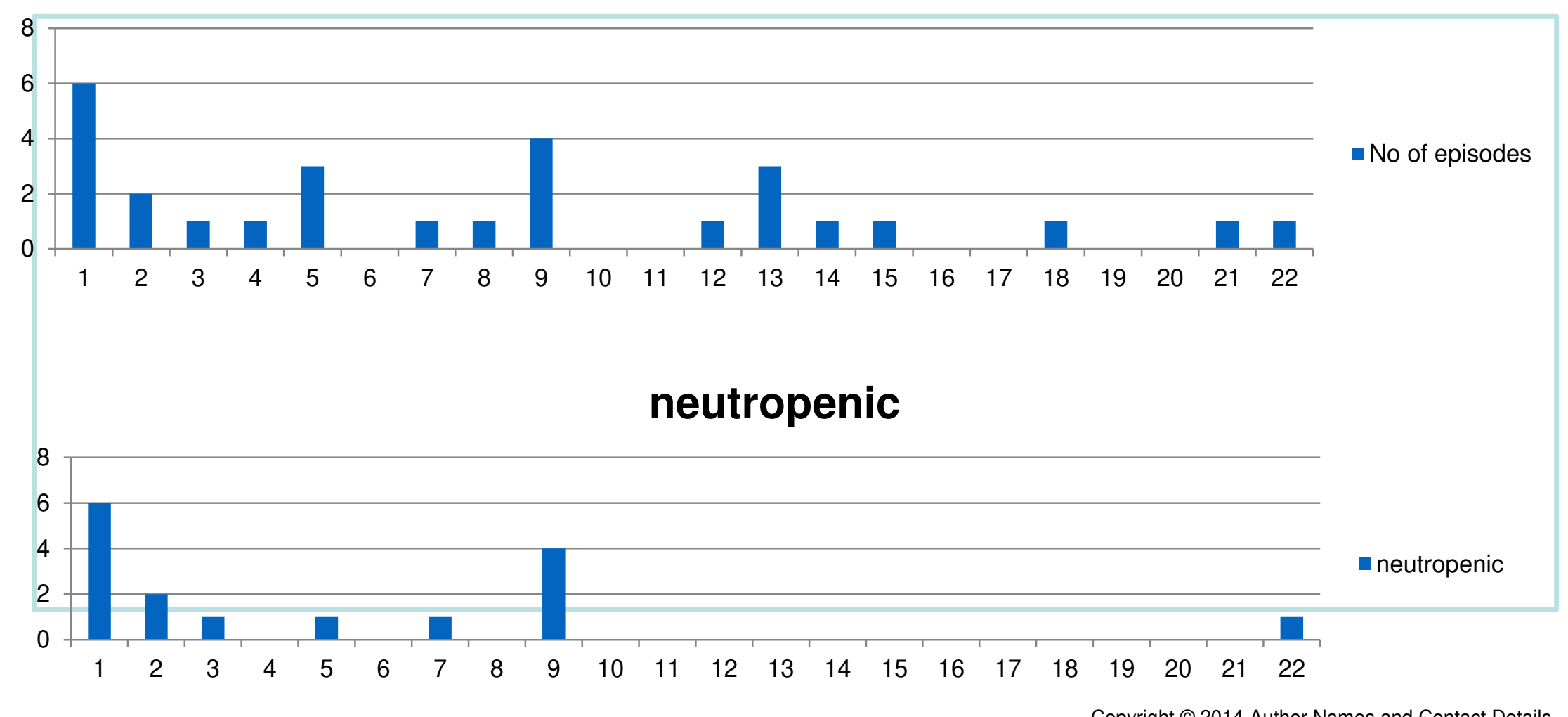

